\title{
Avaliação da Qualidade de Vida e da prevalência de risco de sobrepeso e obesida de em moradores da zona urbana de Milagres - CE
}

\author{
João Paulo da Silva Maciel ${ }^{1}$; Thiago Cabral de Souza ${ }^{2}$; João Marcos Ferreira de Lima e Silva ${ }^{3}$; \\ Plínio Pinto Malveira ${ }^{4}$; Raissa Araújo Rolim ${ }^{5}$
}

\begin{abstract}
Resumo: O objetivo do estudo foi analisar a qualidade de vida, prevalência de sobrepeso e obesidade em moradores da zona Urbana. A população foi composta por moradores da zona urbana de milagres $-\mathrm{CE}$ de ambos os sexos. A amostra para este estudo foi de 100 habitantes, sendo 47 do sexo masculino e 53 do sexo feminino, com idades entre 14 e 70 anos residentes na zona Urbana do Município de Milagres, Estado do Ceara. O instrumento utilizado na coleta dos dados foi o Pentáculo do Bem-estar, que abrange os componentes relacionados à nutrição, atividade física, comportamento preventivo $(\mathrm{CP})$, relacionamentos e controle do estresse (CE). Para análise do estado nutricional foi utilizado o Índice de Massa Corporal (IMC) e a Relação CinturaQuadril (RCQ). Utilizou-se de estatística descritiva, por meio do software SPSS versão 15.0. Os resultados mostram que, $14,9 \%$ dos homens e $17,0 \%$ das mulheres apresentam risco baixo e $36,2 \%$ dos homens e $24,5 \%$ das mulheres apresentam risco moderado, $29,8 \%$ dos homens e $35,8 \%$ das mulheres, apresentam risco alto; e $19,1 \%$ dos homens e $22,6 \%$ das mulheres, risco muito alto. Conclui-se que há predominância de escores negativos na análise da Qualidade de Vida, tais fatores podem ter influenciado na prevalência de Sobrepeso e Obesidade. Sugere-se maior controle nutricional e pratica de exercícios físicos, melhorias nos hábitos alimentares, visando prevenir os níveis elevados de gordura corporal.
\end{abstract}

Palavras chaves: qualidade de vida, sobrepeso e obesidade.

\section{Evaluation of Quality of Life and the risk of overweight and obesity prevalence in residents of urban zone Milagres - CE}

\begin{abstract}
The objective of the study was to assess quality of life, prevalence of overweight and obesity in residents of Urbana area. The population consisted of residents of the urban area of miracles - CE of both sexes. The sample for this study was 100 inhabitants, 47 males and 53 females, aged between 14 and 70 years living in the urban area of the City of Milagres, Ceara State. The instruments used in data collection was used the Pentacle Welfare, covering components related to nutrition, physical activity, preventive behavior (CP), relationships and stress management (EC). For analysis of the nutritional status was determined with body mass index (BMI) and Waist-Hip Ratio (WHR). We used descriptive statistics, using the SPSS software version 15.0. The results show that $14.9 \%$ of men and $17.0 \%$ women and $36.2 \%$ lower risk of men and $24.5 \%$ of women at moderate risk, $29.8 \%$ of men and $35.8 \%$ of women at high risk; and $19.1 \%$ of men and $22.6 \%$ women, very high risk. It follows that there is a predominance of negative scores in the analysis of quality of life, such factors may have influenced the prevalence of overweight and obesity. Suggests a higher nutritional control and practice of physical activity, improvements in eating habits in order to prevent high levels of body fat.
\end{abstract}

Key words: quality of life, overweight and obesity.

\footnotetext{
${ }^{1}$ Graduado em Licenciatura em Educação Física pela Faculdade Leão Sampaio.

${ }^{2}$ Formado em Educação Física no Centro Universitário UNILEÃO, Juazeiro do Norte Ceará. Coordenador Técnico e Diretor da Escola de Futebol de Base do Cruzeiro Esporte Clube de Minas Gerais núcleo Juazeiro do Norte, a Cruzeiro Cariri. Pós-Graduando em Fisiologia do Exercício e Grupos Especiais, no Centro Universitário UNILEÃO. Autor correspondente: cabral.10@gmail.com

${ }^{3}$ Graduado em Licenciatura Plena em Educação Física pela Universidade Federal da Paraíba. Mestrado em Educação Física pela Universidade Federal de Santa Catarina. Docente no Centro Universitário UNILEÃO, Juazeiro do Norte Ceará e na Faculdade Estácio de Medicina de Juazeiro do Norte - CE.

${ }^{4}$ Médico pela Universidade Federal do Ceará - UFC. Email: plinio_malveira@ hotmail.com;

${ }^{5}$ Médica pela Universidade Federal do Ceará - UFC. E-mail: raissa_rolim@ @otmail.com.
} 
Id on Line Revista Multidisciplinar e de Psicologia

Id on Line Multidisciplinary Journal and Psycology

\section{Introdução}

No Brasil, a prevalência de obesidade em adultos também pode ser considerada um problema de saúde pública. Com a modernidade e o avanço tecnológico, as pessoas em geral, vêm se tornando mais sedentárias e negligenciando vários fatores relacionados ao melhor estado de saúde. Esses fatores são citados como sendo, a nutrição, atividade física, comportamento preventivo, relacionamentos e stress, e mostram-se significativamente associados com a longevidade, e o melhor padrão de qualidade de vida (NAHAS, 2003).

Atualmente a Saúde é entendida não apenas como o estado de ausência de doenças, mas, numa perspectiva mais holística, a saúde é considerada como uma condição humana com dimensões física, social e psicológica, caracterizada num contínuo, com pólos positivos e negativos onde, no pólo positivo estão os comportamentos relacionados à saúde, e no pólo negativo os comportamentos de risco e a morte.

Ao analisar o conceito de saúde da OMS, "como um completo estado de bem-estar físico, mental e social e não apenas a ausência de doença o u enfermidade, reafirmam suas crenças, com as quais concordo, de que a saúde das pessoas deve ser encarada como "um assunto ligado às próprias pessoas (DEJOURS, 1986) "alheia a qualquer padronização e a qualquer determinação fixa e pré-estabelecida (CAPONI, 1997), de modo a impedir diferentes interpretações e legitimações, a priori, de controle e governo dos outros.

No Brasil, as mudanças demográficas, socioeconômicas e epidemiológicas ao longo do tempo permitiram que ocorresse a denominada transição nos padrões nutricionais, com a diminuição progressiva da desnutrição e o aumento do sobrepeso e obesidade (NAHAS, 2003). Assim, o aumento do sobrepeso e da obesidade, vem se tornando um dos maiores problemas de saúde das sociedades contemporâneas, o excesso de gordura está relacionado ao surgimento de várias doenças, principalmente pelo avanço de forma rápida e progressiva sem diferenciar raça, sexo, idade ou nível social.

Considerando as informações, os índices de sobrepeso e obesidade têm crescido de forma assustadora em diversos países industrializados e em desenvolvimento, o que tem tornado o controle da quantidade de massa gorda, uma das principais preocupações de vários órgãos de saúde pública (ORGANIZAÇÃO, 2003). 
Desta maneira, cada vez mais a obesidade vem chamando a atenção da comunidade científica, por se mostrar uma doença grave, multifacetada e de genética complexa (BRASIL, 2002). O Instituto Brasileiro de Geografia e Estatística (AMORIM; SILVA; DANTAS; FERNANDES, 2004) em pesquisa de orçamentos familiares (POF) constataram que o excesso de peso já atinge 38,8 milhões de brasileiros adultos, dos quais 10,5 milhões são considerados obesos. Outro fato importante observado na pesquisa, é que nas áreas rurais, o excesso de peso já representa $21 \%$ na região Nordeste, $40 \%$ no Sul, ficando entre 28 e $34 \%$ nas demais regiões rurais.

Em um estudo sociológico, ressalta que desde os anos 70 a modernização forçada do campo e o desenvolvimento tendencioso e excludente, vêm ocasionando mudanças no perfil do estilo de vida das populações rurais repercutindo em grandes transformações sociais, culturais e econômicas (MARTINS, 2001).

Com base no exposto e a par das evidências de que o homem contemporâneo utiliza-se cada vez menos de suas potencialidades corporais, e que este fato é decisivo na má qualidade de vida e na aquisição de várias patologias crônicas, o presente estudo, objetiva analisar a qualidade de vida, prevalência de sobrepeso e obesidade em moradores de ambos os sexos da zona Urbana do município de Milagres no estado do ceara.

\section{Material e Métodos}

Trata-se de uma pesquisa de uma pesquisa quantitativa, descritiva exploratória de campo com delineamento do tipo estudo de caso.

“A pesquisa quantitativa considera o que pode ser quantificável, o que significa traduzir em números opiniões e informações para classificá-las e analisá-las (MARCONI; LAKATOS, 2010)".

Marconi e Lakatos (2010, p. 187) definem também que: [...] A Pesquisa de campo é aquela utilizada com o objetivo de conseguir informações e/ou conhecimentos acerca de um problema, para o qual se procura uma resposta, ou uma hipótese, que se queira comprovar, ou, ainda, descobrir novos fenômenos ou as relações entre eles.

Para Thomas e Nelson (2002), a pesquisa descritiva é realizada através da observação, análise e descrição objetivas e completas de um fenômeno. 
Já a pesquisa exploratória "[...] busca a maior familiaridade com a questão do problema envolvendo um levantamento bibliográfico através de entrevistas com pessoas que tiveram que buscar e obter informações com o problema pesquisado". (VERGARA, 2003, p.28).

O estudo de Caso oferece uma caraterística generalista, uma vez que não proporciona o conhecimento preciso das características de uma população e sim uma visão global de determinado problema (MARCONI; LAKATOS, 2010, p. 187).

Para o referido estudo a população foi composta por moradores da zona urbana de milagres - CE de ambos os sexos. A amostra para este estudo foi de 100habitantes, sendo 47 do sexo masculino e 53 do sexo feminino, com idades entre 14 e 70 anos residentes na zona Urbana do Município de Milagres, Estado do Ceara. Foi utilizado com critérios de inclusão indivíduos com idade entre 14 e 70 anos, residirem na cidade de milagres - CE e aceitarem participar da pesquisa mediante autorização, através do termo de consentimento livre esclarecido (TCLE), os critérios de exclusão foi descumprir todos os critérios acima citados.

Os sujeitos foram selecionados pelo método não probabilístico acidental e por conglomerado, de modo que a amostra foi composta por um subconjunto da população Urbana, formada pelos sujeitos que se disponibilizaram em participar do estudo.

Para a avaliação antropométrica: resultantes das medidas de massa corporal e estatura, utilizou-se a balança digital da marca Camry, (capacidade de $150 \mathrm{~kg}$ e divisão em $100 \mathrm{~g}$ ) e estadiômetro $(200 \mathrm{~cm})$ da marca Sanny, respectivamente; O Índice de Massa Corporal (IMC) foi calculado mediante divisão do peso da massa corporal pelo quadrado da estatura.

A Relação Cintura-Quadril (RCQ) foi calculada mediante a divisão dos perímetros de cintura e quadril, medidos por meio de uma fita antropométrica da marca Sanny (com resolução de $150 \mathrm{~cm}$ ).

Para avaliar a qualidade de vida (QV): Utilizou-se o questionário do Pentáculo do Bem-estar, considerando aspectos relacionados à nutrição (NUT), atividade física (AF), comportamentos preventivos (CP), relacionamentos (REL). E controle do estresse (CE). Para tanto, considerou-se a seguinte escala: 0 e 1,9 = escore negativo, 2 e $3=$ escore positivo.

Os procedimentos constituíram-se dentro dos padrões éticos legais referentes à pesquisa com seres humanos da resolução 446/12. Seguindo a seguinte ordem: Inicialmente foi feito contato e pedido a autorização para a participação no estudo, mediante a assinatura 
de um Termo de Consentimento Livre e Esclarecido de acordo com o Ministério da Saúde. Procedeu-se a coleta com a realização da avaliação antropométrica e avaliação da qualidade de vida.

Foram usadas as estatísticas descritivas e as informações foram tabulados por meio do programa Microsoft Office Excel 2007 e transportados para o programa SPSS, versão 15, para exploração completa dos dados (média, valores mínimos, máximos e desvio-padrão (DP) e percentual). Logo após levado para programa Microsoft Office Word 2007.

\section{Resultados e Discussões}

Os dados referentes aos resultados desta pesquisa abrangendo a qualidade de vida e da prevalência de sobrepeso em moradores da zona urbana de milagres-CE, foram apresentados em forma tabelas a fim de demonstrar estatisticamente valores referentes a analise dos dados obtidos.

Ao final da coleta de dados, devido em grande parte dos moradores e ao número de pessoas existentes no município, não obtivemos evasões e perdas, desta forma chegou-se ao total de $0,0 \%$ de perdas, sendo que, em nenhum dos estratos este número superou o valor estimado no cálculo amostral.

Tabela 1. Distribuição de Mínimo, máximo, média e percentual dos indicadores antropométricos e de composição corporal $(\mathrm{N}=100)$.

\begin{tabular}{|c|c|c|c|c|c|c|c|c|}
\hline \multirow[t]{2}{*}{ Variáveis } & \multicolumn{4}{|c|}{ Masculino $(n=47)$} & \multicolumn{4}{|c|}{ Feminino $(n=53)$} \\
\hline & Mín.* & Máx.** & $\mathbf{X}^{* * *}$ & $\% * * * *$ & Mín.* & Máx.** & $\mathbf{X}^{* * *}$ & $\% * * * *$ \\
\hline Idade (anos) & 14 & 70 & 29,1 & 52,2 & 14 & 70 & 26,7 & 47,8 \\
\hline $\begin{array}{c}\text { Massa Corporal } \\
(\mathrm{kg})\end{array}$ & 40,0 & 88,0 & 66,3 & 50,2 & 46,2 & 94,0 & 65,7 & 49,8 \\
\hline Estatura (m) & 1,5 & 1,9 & 1,7 & 51,5 & 1,5 & 1,8 & 1,6 & 48,5 \\
\hline Cintura & 55,0 & 54,0 & 68,1 & 51,1 & 91,0 & 69,0 & 65,3 & 48,9 \\
\hline Quadril & 66,0 & 81,0 & 91,8 & 51,1 & 107,0 & 101,0 & 87,8 & 48,9 \\
\hline IMC (kg/m2) & 16,1 & 31,9 & 23 & 48,9 & 19,3 & 31,0 & 24 & 51,1 \\
\hline RCQ & 0,74 & 0,98 & 0,89 & 51,4 & 0,78 & 0,89 & 0,84 & 48,6 \\
\hline
\end{tabular}

*Mín. - Mínimo - **Máx. Máximo - ***X - Média - ****\% - Percentual / Fonte: Pesquisa 2013. 
De acordo com a tabela 01 pode-se observar a análise descritiva da amostra utilizada para o referido trabalho. Destacando os resultados adquiridos pela amostra com mínimo, máximo media e percentual.

Verificou-se que em relação à idade que idade mínima entre os indivíduos pesquisados foi de (14,0 anos) para o sexo masculino e feminino. Ao que se refere à idade máxima foi de (70 anos) para ambos os sexos, com media de idade de (29,1 anos) equivalente ao percentual de52,2\%para o sexo masculinoe media de $(26,7$ anos) equivalente a $(47,8 \%)$ para o sexo feminino.

$\mathrm{Na}$ variável massa corporal pode-se observar que o valor mininoobtidoentre os indivíduos pesquisados foi de $(40,0 \mathrm{~kg})$ para o sexo masculino e $(46,2 \mathrm{~kg})$ feminino. Ao que se refere o peso máximofoi de $(88,0 \mathrm{~kg})$ para o sexo masculino e $(94,0 \mathrm{~kg})$ para o sexo feminino, a media obtida foi de $(66,3 \mathrm{~kg})$ equivalente ao percentual de $(50,2 \%)$ para o sexo masculino e media de $(65,7 \mathrm{~kg})$ equivalente a $(49,8 \%)$ para o sexo feminino.

Outro avaliado como descrito na tabela foi à estatura não tendo nenhuma diferença entre os valores mínimos dos sexos masculino e feminino (1,5 m). já para os valores máximos a diferença não foi tão significante, visto que o valores obtidos foram de (1,9 m)para o sexo masculino e $(1,8 \mathrm{~m})$ para sexo feminino, com media de $(1,7 \mathrm{~m})$ equivalente ao percentual de $(51,5 \%)$ para o sexo masculino e $(1,6 \mathrm{~m})$ equivalente ao percentual de $(48,5 \%)$ para o sexo feminino.

Em relação à descriminação dos resultados da cintura, a mínima para o sexo masculino foi de $(55,0 \mathrm{~cm})$ e para o sexo feminino foi de $(54,0 \mathrm{~cm})$. Já a máxima foi de $(91,0 \mathrm{~cm})$ para o sexo masculinoe $(69,0 \mathrm{~cm})$ para o sexo feminino, com média de $(68,1 \mathrm{~cm})$, equivalente a $(51,1 \%)$ para o sexo masculino e de $(65,3 \mathrm{~cm})$, equivalente a $(48,9 \%)$ para o feminino. Desse modo levando em consideração apenas a média geral, observamos que não existe uma discrepância muito elevada entre os gêneros.

Com relação a variável quadril, pôde-se perceber que a média mínima para o sexo masculino de $(66,0 \mathrm{~cm})$ foi bem menor em comparação ao sexo feminino, cerca de $(81,0 \mathrm{~cm})$. O valor máximo foi de $(107,0 \mathrm{~cm})$ para o sexo masculinoe de $(101 \mathrm{~cm})$ para o feminino, observamos que neste critério a relação cintura-quadril foi bem maior para o sexo masculino. Em contra partida, fazendo a média geral, o sexo feminino obteve um índice mais alto, cerca 
de $(91,8 \mathrm{~cm})$ equivalente a $(51,1 \%)$ para o sexo masculino, já o sexo oposto, a média foi de $(87,8 \mathrm{~cm})$ equivalente $\mathrm{a}(47,8 \%)$.

Os resultados exposto nesta tabela demonstra que em média a amostra estudada é formada por pessoas adultas jovens. De forma que os homens mostram-se mais altos e pesados em relação às mulheres.

Desta forma em média, ambos os sexos estão com o IMC normal, sem constatação de risco a saúde. Em relação ao RCQ a interpretação dos resultados obtidos, tanto nos homens quanto nas mulheres mostrou níveis moderados de riscos para desenvolver doenças crônicas metabólicas.

Tabela 2. Valores de média e Desvio Padrão dos componentes, nutrição, atividade física, comportamento preventivo, relacionamentos e controle do stress de ambos os sexos $(\mathrm{N}=100)$.

\begin{tabular}{c|c|c}
\hline VARIÁVEIS & $\mathbf{X}^{*}$ & $\mathbf{D} \mathbf{p}^{* *}$ \\
\hline Nutrição "A" & & \\
Nutrição "B" & 2,2 & 1,1 \\
Nutrição "C" & 1,8 & 0,7 \\
Atividade Física "D", & 2,6 & 0,0 \\
Atividade Física "E" & 1,7 & 0,6 \\
Atividade Física "F" & 1,1 & 0,4 \\
Comportamento preventivo "G" & 2,1 & 1,0 \\
Comportamento preventivo "H" & 0,4 & 0,0 \\
Comportamento preventivo "I" & 1,8 & 0,7 \\
Relacionamentos "J" & 2,9 & 1,9 \\
Relacionamentos "K" & 2,4 & 1,4 \\
Relacionamentos "L" & 1,8 & 0,7 \\
Controle do stress "M" & 2,0 & 1,0 \\
Controle do stress "N" & 2,7 & 1,7 \\
Controle do stress "O" & 0,8 & 0,1 \\
\hline
\end{tabular}

*X - Média - **Dp - Desvio Padrão / Fonte: Pesquisa 2013.

Os dados adquiridos que estão expostos na Tabela 2, estão relacionados às médias dos escores dos componentes da qualidade de vida. O componente da Nutrição obteve no item B escores médios negativos $(1,8)$; o envolvimento com a pratica de Atividade Física mostrou escores negativos nos itens D $(1,7)$ e E $(1,1)$; O Comportamento Preventivo mostrou escore negativo na questão $\mathrm{G}(0,4)$ e H $(1,8)$; Na questão relacionada ao Relacionamento, os sujeitos 
Id on Line Revista Multidisciplinar e de Psicoloqia

Id on Line Multidisciplinary Journal and Psycology

investigados mostraram média nos os itens $\mathbf{J}(2,4)$ e $\mathrm{L}(2,0)$ positivos. Na observação do Controle do Stress constatou-se escore negativo na questão N $(0,8)$.

Santos (2004) investigou o estilo de vida, a percepção e a concepção de qualidade de vida de moradores da zona urbana no estado de São Pauloe constatou que, apesar dos mesmos terem consciência de que a saúde é o alicerce fundamental da qualidade de vida, eles pouco se preocupam com os comportamentos de risco a saúde dando mais atenção, nesta fase, as questões de acumulação de bens materiais, o que, segundo eles, justificaria sacrifícios de ordem pessoal para alcançar objetivos materiais.

Diversas pesquisas apontam que os comportamentos de risco adquiridos durante a fase universitária, tendem a se perpetuar durante a fase profissional (adulta), além disso, as condutas de saúde estabelecidas durante o período da adolescência e juventude podem ter impacto significante na ocorrência de doenças futuras. Estudo europeu tem demonstrado que jovens tendem a adotar um número maior de condutas de risco á saúde (VON AH et. al., 2004; NAHAS, 2006).

Tabela 3. Análise dos níveis de sobrepeso e obesidade considerando o IMC dos moradores de ambos os sexos da zona Urbana de Milagres - CE, $(\mathrm{N}=100)$.

\begin{tabular}{c|c|c|c|c}
\hline Classificação IMC & \multicolumn{2}{|c|}{ Feminino } & \multicolumn{2}{c}{ Masculino } \\
\hline & $\mathbf{N}^{*}=\mathbf{4 7}$ & $\mathbf{\%} * *=\mathbf{1 0 0}$ & $\mathbf{N} * \mathbf{5 3}$ & $\mathbf{\%} * * \mathbf{1 0 0}$ \\
Peso abaixo do ideal & - & - & 03 & 5,7 \\
Peso Normal & 32 & 68,1 & 35 & 66,0 \\
Sobrepeso & 14 & 29,8 & 14 & 26,4 \\
Obesidade & 01 & 2,1 & 01 & 1,9 \\
& & & & \\
\hline
\end{tabular}

*N - Quantidade - **\% - Percentual / Fonte: Pesquisa 2013.

Conforme a tabela 3 acima dados referentes à análise dos níveis de sobrepeso e obesidade, considerando o IMC. Observou-se que 5,7\% das mulheres apresentaram está com Peso abaixo do ideal, $68,1 \%$ dos homens peso normal, seguido de $66,0 \%$ nas mulheres, houve uma prevalência de sobrepeso, $29,8 \%$ nos homens, e $26,4 \%$ nas mulheres, e $2,1 \%$ de obesidade grau I nos homens, e 1,9\% nas mulheres.

O IMC tem sido utilizado em vários estudos para determinar a composição corporal de indivíduos de ambos os sexos, apontando tanto para a obesidade quanto para o baixo peso, encontrou-se uma prevalência de sobrepeso ou obesidade referente aos demais níveis 
Id on Line Revista Multidisciplinar e de Psicoloqia

Id on Line Multidisciplinary Journal and Psycology

considerando a distribuição da gordura corporal, ou a prevalência de obesidade andróide gordura localizada na região central ou visceral(MARTINS, 2001).

Tabela 4. Análise dos níveis de sobrepeso e obesidade considerando a RCQ dos moradores de ambos os sexos da zona urbana de milagres. $(\mathrm{N}=100)$.

\begin{tabular}{c|c|c|c|c}
\hline \multicolumn{2}{c}{ Classificação RCQ } & \multicolumn{2}{c}{ Masculino } & Feminino \\
\hline & $\mathbf{N *}=\mathbf{4 7}$ & $\mathbf{\%} * *=\mathbf{1 0 0}$ & $\mathbf{N} * \mathbf{5 3}$ & $\mathbf{\%} * \mathbf{*}=\mathbf{1 0 0}$ \\
& 07 & 14,9 & 09 & 17,0 \\
Baixo & 17 & 36,2 & 13 & 24,5 \\
Moderado & 14 & 29,8 & 19 & 35,8 \\
Alto & 09 & 19,1 & 12 & 22,6 \\
Muito Alto & & & & \\
\hline
\end{tabular}

*N - Quantidade - **\% - Percentual / Fonte: Pesquisa 2013.

De acordo a Tabela 4 acima os resultados mostram que, 14,9\% dos homens e 17,0\% das mulheres risco baixo e 36,2\% dos homens e $24,5 \%$ das mulheres apresentam risco moderado, 29,8\% dos homens e 35,8\% das mulheres, apresentam risco alto; e 19,1\% dos homens e $22,6 \%$ das mulheres, risco muito alto.

De acordo com a tabela de classificação Bray e Gray, pôde-se observar neste último gráfico que, a maioria da amostra pesquisada apresentou baixo risco para desenvolver doenças cardiovasculares, obesidade e hipertensão.

Em relação ao risco moderado, teve-se uma diferença bastante relevante entre os sexos, com esse achado pode-se perceber que os homens possuem maior quantidade de massa corpórea do que às mulheres, ou seja, as mesmas apresentaram um índice considerável de gordura localizada, o que as levam a ter maior predisposição para as doenças citadas anteriormente.

A RCQ parece ser um ótimo indicador para predição de desenvolvimento de doenças metabólicas crônicas, também é elemento de classificação da obesidade (COSTA, 2001).

A prática regular de atividade física apresenta uma relação inversa com risco de doenças crônicas degenerativas e tem um efeito positivo na qualidade de vida e em outras variáveis psicológicas. Para tanto, tem sido dada ênfase à redução do sedentarismo, mediante planos de adoção de atividade física regular para melhoria da saúde individual e coletiva. 
A prática regular de atividade física contribui consideravelmente para evitar as doenças crônicas degenerativas, em razão de uma série de alterações fisiológicas e psicológicas e por diminuir as despesas com tratamentos e os índices de afastamento do trabalho (LIMA, 2003).

\section{Conclusões}

Considerando o objetivo do estudo, a amostra e os resultados do presente trabalho, conclui-se que: neste estudo o nível de atividade física diária mostrou estar significativamente associado à prevalência de sobrepeso e obesidade. Embora esse resultado deva ser avaliado com cautela, devido à falta de validação do instrumento de coleta de dados, ele pode ser utilizado como indicativo do perfil de atividade física assumida pela populaçãoem estudo.

Estudos transversais com adultos têm mostrado uma relação de causalidade reversa entre atividade física e obesidade. Entretanto, as medidas necessárias para a sua solução são tomadas, entre elas, a inclusão de um programa de exercícios físicos na rotina.

A maior parte dos fatores da qualidade de vida estudada apresenta escores negativos, tais comportamentos podem ter contribuído para a prevalência de sobrepeso e obesidade encontrados na amostra. Assim, sugere-se uma maior conservação de hábitos alimentares adequados, prática regular de atividade física, aquisição de comportamento preventivo e controle do estresse, visando prevenir e controlar os níveis de sobrepeso e obesidade na população Urbana do município de Milagres - CE.

Concluir se que o método índice de massa corporal(IMC) eRelação Cintura-Quadril (RCQ) utilizados nestes estudo é um indicador antropométrico bem simples, que auxilia na identificação de certas doenças e portanto concluiu-se com os dados analisados uma relação considerável de risco a saúde no que se refere risco moderado do ambos os sexos, demonstrando uma alerta para o sobrepeso e doenças crônico-degenerativas.

Nesse estudo foi demonstrado que os componentes da avaliação da qualidade de vida de relacionada a nutrição, controle do stress e atividade física do constructo estilo de vida individual dos moradoresda zona urbana de Milagres - CE, apresentam deficiências preocupantes e precisam ser abordados por intervenções educacionais paralelas com 
Id on Line Revista Multidisciplinar e de Psicoloqia

Id on Line Multidisciplinary Journal and Psycology

responsabilidade interdisciplinar. Conclui-se que há predominância de escores negativos na análise da Qualidade de Vida, tais fatores podem ter influenciado na prevalência de Sobrepeso e Obesidade. Sugere-se maior controle nutricional e pratica de exercícios físicos, melhorias nos hábitos alimentares, visando prevenir os níveis elevados de gordura corporal.

No entanto para que se possam obter resultados com maior prevalência sugerem-se a realização de outros estudos que sobre avaliação da qualidade de vida e da prevalência de sobrepeso em moradores da zona urbana de milagres - CE, na busca de obter uma associação de comportamentos inadequados de alimentação e atividade física que assim podem resultar em níveis reduzidos de controle de estresse que poderão desencadear um baixo rendimento, promovendo assim o avanço do conhecimento relacionado a esta temática na área da saúde.

\section{Referências}

AMORIM, P.R.S; SILVA, S.C; DANTAS E.H. M; FERNANDES, F.J. Sensibilidade e especificidade do índice de massa corpórea na determinação da obesidade. Um estudo em brasileiros de ambos os sexos. Journal Fitness e Performance, p. 71-75, 2004.

BRASIL, Ministério da Saúde. Conselho Nacional de Saúde. Manual operacional para comitês de ética em pesquisa. Brasília DF: Ministério da Saúde; 2002.

CAPONI, S. A proposito Del concepto de salud. Florianópolis: Mimeogr. UFSC, 1997.

COSTA, R.F. Composição corporal teoria e prática da avaliação. São Paulo: Manole; 2001.

DEJOURS, C. Por um novo conceito de saúde. Revista Brasileira de Saúde Ocupacional, São Paulo, v.14, n.54, p.7-11, abr./ jun.1986.

LIMA, V. Ginástica laboral: atividade física no ambiente de trabalho. São Paulo: Phorte, 2003.

MARTINS, J.S. O futuro da sociologia rural e sua contribuição para a qualidade de vida rural. Revista Ciência e Cultura, p. 31-36, 2001.

NAHAS, M.V. Atividade física, saúde e qualidade de vida: conceitos e sugestões para um estilo de vida ativo. 3. Ed. Londrina: Midiograf; 2003. 
ORGANIZAÇÃO, Pan-Americana da Saúde. Doenças crônico-degenerativas e obesidade: Estratégia mundial sobre alimentação saudável, atividade física e saúde. Brasília, DF: Formatos Design; 2003.

SANTOS, H.H. Manual prático para elaboração de projetos, monografias, dissertações e teses na área de Saúde. João Pessoa: Universitária; 2004.

SANTOS, J.F.S.Estilo de vida e percepção de qualidade de vida de acadêmicos de ciências tecnológicas: um estudo de caso. In: Anais do II congresso cientifico latino-americano da FIEP. Foz do Iguaçu: FIEP, 2004.

VON AH, D.; EBERT, S.; NGAMVITROJ, A.; PARK, N.; KANG, D.H. Predictors of health behaviours in college students. Journal Adventure Nurse,p. 463-74, 2004.

\section{Como citar este artigo (Formato ABNT):}

MACIEL, J.P.S.; SOUZA, T.C.; SILVA, J.M.F.L.; MALVEIRA, P.P.; ROLIM, R.A. Avaliação da Qualidade de Vida e da prevalência de risco de sobrepeso e obesida de em moradores da zona urbana de Milagres - CE. Id on Line Revista Multidisciplinar e de Psicologia, Set-Out de 2016, vol.10, n.31, Supl 2, p. 318-329. ISSN 19811179 .

Recebido: 25/09/2016

Aceito: 28/09/2016 